\title{
POLYPHARMACY INDUCED DRUG INTERACTIONS, ADVERSE DRUG REACTIONS (ADR) AND MEDICATION ERRORS IN TERTIARY CARE SOUTH INDIAN HOSPITAL
}

\author{
SIDDARAMA R. , BHARATH NAIDU J., JOSHISREE K. P., SAHITHI LAKSHMI V. \\ Department of Pharmacy Practice, Creative Educational Society's College of Pharmacy, N. H.4, Chinnatekur, Kurnool, Andhra Pradesh \\ 518218 \\ Email: siddaramaramalingan@gmail.com \\ Received: 04 Sep 2018 Revised and Accepted: 05 Jan 2019
}

\begin{abstract}
Objective: To study the pattern of drug interactions (DI) in our hospital and to identify whether it is associated with polypharmacy. To determine the level of severity of potential drug-drug interactions (PDDI), to detect, monitor and prevention of ADRs in the hospitalized patients and to identify the medication errors (ME).

Methods: A prospective interventional study was conducted in a 300 bedded tertiary care South Indian hospital for a period of 6 mo. Prescriptions were analysed for PDDI using Micromedex software 2.2. The causality and severity of ADRs were assessed by using Naranjo's, WHO UMC Scales and Hart wigs severity scales. ME was identified by review of patient drug charts.

Results: Total 190 prescriptions were analyzed, in which 1028 drug interactions were seen. Out of which 718 were DDI, 198 DFI, 100 DEI, and 12 DTI were observed. More number of DI was seen in cardiovascular drugs, antibiotics followed by antacids and antiulcer agents. A total of 52 ADRs were identified in 43 patients. Diuretics, cardiovascular drugs were associated with a higher incidence of ADRs followed by Anti-Diabetic agents. 58 ME was seen in 190 prescriptions, among them omission error, prescribing errors and Wrong dose error was seen.
\end{abstract}

Conclusion: Clinical pharmacist plays a potential role in the health care system in assisting the physician i.e. modifying the number of drugs taken, number of doses taken, medication adherence, identification of drug interactions, preventing, monitoring and detection of ADRs and identifying the medication errors.

Keywords: Drug interactions, Adverse drug reactions, Medication Error, Micromedex, Clinical Pharmacist

(C) 2019 The Authors. Published by Innovare Academic Sciences Pvt Ltd. This is an open-access article under the CC BY license (http://creativecommons.org/licenses/by/4.0/) DOI: http://dx.doi.org/10.22159/ijpps.2019v11i2.29508

\section{INTRODUCTION}

The definition of polypharmacy in the literature is not uniform. However, the word 'poly' is a Greek word and means many or much. There are basically two approaches to the definition [1]. Analysis of prescription data could provide the bases for reviewing prescriptions practices and developing measures to promote the rational use of drugs and prevent drug-related problems. According to the $\mathrm{WHO}$, the rational use of drugs requires that patients receive medications appropriate to their clinical needs, in doses that meet their own individual requirements, for an adequate period of time, and at the lowest cost to the and their community [2]. Furthermore, attention should be paid to the fact that the body of the older adults presents changes in their physiological functions that may lead to differentiated pharmacokinetics and greater sensitivity to both therapeutic and adverse drug effects. Pharmacokinetics, pharmacodynamics, and clinical outcomes are affected by a number of patient-specific factors, including age, sex, ethnicity, genetics, disease processes, polypharmacy, drug dose, and frequency, social factors, and many other factors [3]. Specifically, the burden of taking multiple medications has been associated with greater health care costs and an increased risk of adverse drug events (ADEs), drug interactions, medication non-adherence, reduced functional capacity and multiple geriatric syndromes [4]. The aim of the study was to evaluate the drug interactions (DIs), to assess, monitor and report the adverse drug reactions (ADRs) and to minimize medication errors.

During hospitalisation, a frequency of major and moderate drugdrug interactions per patient was 1.11, which was higher compared to hospital admission or to hospital discharge. Incidence of potential drug-drug interactions was very high almost in $89 \%$ of all prescriptions. The prevalence of ADR's at admission was $12.4 \%$ and ADR's causing admission was $8.1 \%$. Overall incidence of serious ADR's was $6.7 \%$ and of fatal ADR's was $0.32 \%$ of hospitalized patients. Prescribing errors are the most frequent subtype of medication errors, occurring in $7 \%$ medication orders, $50 \%$ of hospital admissions and $2 \%$ of inpatients. ADR's remain a significant contributing factor to morbidity and mortality. There is a definite need to further understand the incidence and prevalence of ADR's in an ambulatory population.

By this type of study, we can give individualised treatment to each patient that increase the quality of life and further expect outcomes prior to and increasing the safety of drug use.

\section{MATERIALS AND METHODS}

Place of the study: All departments in a 300 bedded tertiary care South Indian Hospital.

Study design: This study is a prospective interventional study using inpatient prescriptions from different departments in a 300 bedded tertiary care South Indian hospital.

Study population: 190 prescriptions were screened

Study duration: 6 mo from September to February

Ethical approval was taken from our hospital Vishwa Bharathi multispecialty hospital, Kurnool, Andhra Pradesh.

Eligibility criteria

Patients are enrolled in the study based on inclusion and exclusion criteria

\section{Inclusion criteria}

Prescription with more than 3 drugs, those who stayed greater than $24 \mathrm{~h}$ in the hospitals was included. Both Age groups greater than 15 $\mathrm{y}$ and the patients who are willing were included in this study. 


\section{Exclusion criteria}

Patients who are uncooperative, not willing to participate in the study were excluded from the study. Pregnancy and lactating women, incomplete patient medical records were excluded. Patients who are consuming other than allopathy medications and those who stayed $<24 \mathrm{~h}$ in the hospitals were excluded. Patients who are diagnosed to have HIV and tuberculosis (TB) and patients who are taking chemotherapy and radiation therapy were excluded from the study.

\section{Study protocol}

A total of 190 prescriptions were selected. Cases and controls were identified by using a computerized DI database system (Micromedex 2.2 version).

\section{Methods for detecting drug interactions}

As research better explains the biochemistry of drug use, fewer ADRs (adverse drug reactions) are Type B and more are Type A.

\section{Methods for detecting adverse drug reactions}

\section{Causality assessment}

A separate data entry format was specially designed including the drug chart, ADRs algorithm, and causality assessment scale. The identification of drug interactions, ADRs and medication errors was done through routine ward round participation and prescription monitoring of hospitalized patients and op patients in all departments. The reports were obtained from health care professionals (nurses, doctors etc). Data were collected from patient's case sheet and transferred to data entry format for evaluation.

\section{Methods of detecting an ADR}

\section{Spontaneous monitoring}

In spontaneous monitoring, treating physician's record suspected ADRs using reporting cards.

\section{Intensive monitoring}

Intensive monitoring is based upon the systematic evaluation of link between the adverse event and drugs involved, patients admitted were followed up until discharged.

Performed screening of patients arriving to a hospital and among them 100 patients taking cardiovascular drugs was included in the study after obtaining inform consent form. The ADRs developed was discussed with the physician and De-challenge or Re-challenge was done based on the ADR severity. The causality and severity assessment were done using Naranjo, WHO-UMC scales, Hart wigs scale and Rawlins and Thompson ADRS respectively.

\section{Methods for detecting medication errors}

The major methods for detecting adverse events are chart review, computerized monitoring, incident reporting, and searching claims data. Medication errors are mainly detected by means of direct observation, voluntary reporting (by doctors, pharmacists, nurses, patients, and others) and chart review and incident reporting.

\section{RESULTS}

The study was conducted in the in-patient department of tertiary south Indian hospital for a period of $6 \mathrm{mo}$. Total drugs used in the 190 prescriptions were 1867 drugs. The average number of drugs per prescription was 9.82 .

\section{Categorisation of polypharmacy}

Table 1: Categorisation of polypharmacy

\begin{tabular}{llll}
\hline S. No. & No of drugs & No of prescriptions & Percentage \\
\hline 1 & Less than 6 & 7 & 3.684 \\
2 & Equal to 6 & 9 & 4.736 \\
3 & More than 6 & 174 & 91.578 \\
& Total & 190 & 100 \\
\hline
\end{tabular}

The total number of prescriptions which contains more than 6 drugs were predominantly high $174(91.57 \%)$ cases whereas $9(4.736 \%)$ prescriptions contains equal to 6 drugs and $7(3.68 \%)$ prescriptions contains less than 5 drugs were shown in table 1.

A total of 185 prescriptions were found to have drug interactions in 190 prescriptions. Out of which $127(69.24 \%)$ were males in which 713 drug interactions were seen, out of 713 drug interactions 482(67.60\%) drug-drug interactions were seen, 119(16.69\%)drugfood interactions were seen, $100(14.02 \%)$ drug-ethanol interactions were seen and 12(1.68\%) drug-tobacco interactions were seen. 315 drug interactions were seen in 63(33.15\%) females, out of which 236(74.92\%) drug-drug interactions were seen, 79(25.07\%) drugfood interactions were seen. Age was found to be an important criterion in the fact that the patient in the age group 51-70 y had experienced maximum drug interactions (in cardiology department).

\section{Classification of drug interactions}

Among that 1028 drug-drug interactions were seen, in which $419(58.35 \%)$ were major, $255(35.51 \%)$ were moderate and $44(6.12 \%)$ were minor.198 drug-food interactions were seen, in which $2(1.01 \%)$ were major, $88(44.44 \%)$ were moderate and 108(54.54\%) were minor.100 drug ethanol interactions were seen, in which $59(59 \%)$ were major, $40(40 \%)$ were moderate. 12 drugtobacco interactions were seen, in which $8(66.66 \%)$ were major, $4(33.33 \%)$ were moderate were shown in table 2 .

Table 2: Severity of drug interactions

\begin{tabular}{|c|c|c|c|c|c|}
\hline S. No. & Severity & DDI & DFI & DEI & DTI \\
\hline 1 & Major & $419(58.35)$ & $2(1.01)$ & $59(59)$ & $8(66.66)$ \\
\hline 2 & Moderate & $255(35.51)$ & $88(44.44)$ & $40(40)$ & $4(33.33)$ \\
\hline \multirow[t]{2}{*}{3} & Minor & $44(6.12)$ & $108(54.54)$ & $1(1)$ & $0(0)$ \\
\hline & Total & $718(100)$ & $198(100)$ & $100(100)$ & $12(100)$ \\
\hline
\end{tabular}

\section{Class wise distribution of drugs}

Antibiotics, Cardiovascular drugs followed with NSAIDS are the major class of drugs with interactions in our study.
In this study antibiotics (13.13\%) and cardiovascular drugs $(13.13 \%)$ are associated with high number of drug interactions followed by antacids(11.11\%), NSAIDS (7.07\%), Corticosteroids (7.07\%), antipsychotics (7.07\%), antidiabetics (6.06\%), 
anticonvulsants (5.05\%), antiplatelet (5.05\%), anticoagulants $(5.05 \%)$, diuretics $(4.04 \%)$, anti TB agent s $(4.04 \%)$, antiasthma agents $(3.03 \%)$, antiemetic's(2.02\%), vitamins and minerals
(2.02\%), anti-malarial(1.01\%), antifungal agents $(1.01 \%)$, antiamoebic $(1.01 \%)$, anti-thyroids agents(1.01\%) and inotropic agents(1.01\%) were shown in fig. 1.

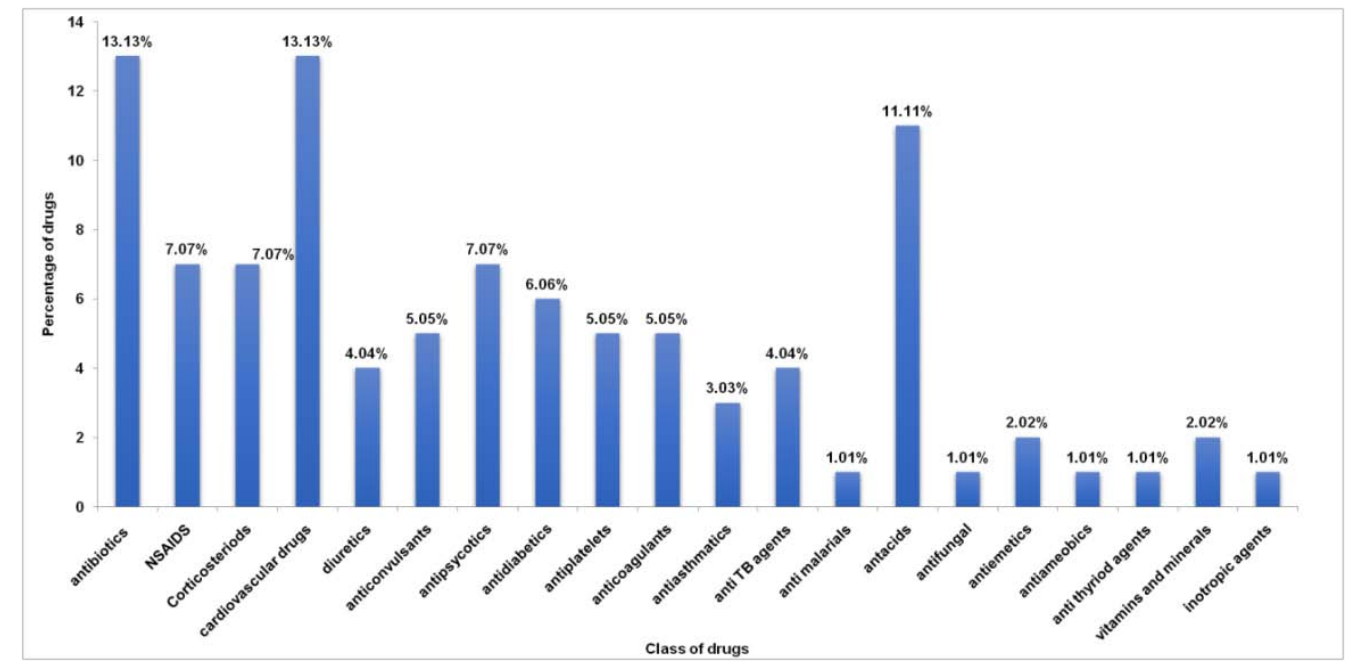

Fig. 1: Class-wise distribution of drugs

\section{Gender wise distribution of ADRs}

Out of 52 ADRs, 36(69.23\%) ADRs were identified in males and $16(30.76 \%)$ ADRs were identified in females. Thus high incidence of ADRs was reported in males. The most vulnerable age group that is prone to ADRs in this study was 61-70 y group of patients.

\section{Severity assessment of ADRs}

The severity assessment of ADRs as mild, moderate, severe was done by using Hart wigs scale. Causality assessment is the method by which the extent of the relationship between the drug and suspected reaction is established. Among different causality scales, the Naranjo's scale and WHO-UMC scale of assessment are most commonly used.

Table 3: Severity assessment of ADRs

\begin{tabular}{lll}
\hline Causality & No of ADRs & \% OF ADRS \\
\hline Mild & 6 & 11.53 \\
Moderate & 38 & 73.07 \\
Severe & 8 & 15.38 \\
Total & 52 & 100 \\
\hline
\end{tabular}

The severity assessment by modified Hart wig's scale showed that 6 (11.53\%) ADRs were mild, 38(73.07\%) ADRs were moderate and 8(15.38\%) ADRs were severe were shown in table 3.

Table 4: Causality assessment of ADRs by Naranjos scale

\begin{tabular}{lll}
\hline Causality & No of ADRs & \% OF ADRS \\
\hline Definite & 10 & 19.23 \\
Probable & 10 & 19.23 \\
Possible & 32 & 61.53 \\
Total & 52 & 100 \\
\hline
\end{tabular}

\section{Causality assessment of ADRs by naranjo's scale}

The assessment by Naranjo's scale showed that out of 52ADRs, $10(19.23 \%)$ ADRs are definite, $10(19.23 \%)$ ADRs are probable and $32(61.53 \%)$ ADRs are possible were shown in table 4.

\section{Causality assessment of ADRs by WHO-UMC scale}

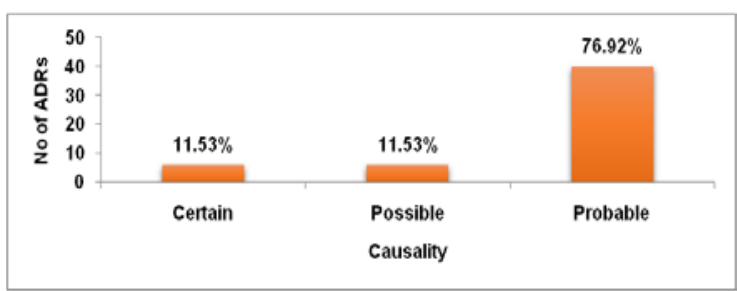

Fig. 2: Causality assessment of ADRs by WHO-UMC scale
The assessment by WHO-UMC scale showed that out of 52ADRs, $6(11.53 \%)$ ADRs were certain, 6(11.53\%) ADRs were possible and 40(76.92\%) ADRs were probable were shown in fig. 2.

\section{Distribution of ADRs based on drug class}

In this study diuretics (28.84\%) and cardiovascular drugs (23.07\%) are associated with a high number of ADRs, followed by antidiabetic agents $(13.46 \%)$.

In this study, diuretics were found to cause ADRs of electrolytic imbalances like hyponatremia-8, hypokalemia-4, hyperkalemia-2, and hypomagnesemia-1. Followed by cardiovascular drugs were found to cause constipation-1, headache- 3 , palpitation-1, dizziness-1, Nausea-1, vomiting-1, cough-2, bloating-2. Antidiabetic agents cause hypoglycaemia-5, CKD-1, hyperkalemia-1. Antibiotics were found to cause ADRs like mild diarrhoea-1, SJ syndrome-1, hypokalemia-1, nausea, and vomiting-1. Anticoagulants cause hypotension-2, sinus bradycardia-1. Anti TB agents cause altered liver enzymes-1, decreased 
appetite-1, dark colour urine-1. Antacids and antiulcer agents cause constipation-2. Anticonvulsants cause sore throat-1, slurred speech-1.
Anticholinergic agents cause psychosis-1. Corticosteroids cause ecchymosis-1 and NSAIDS cause SJ syndrome-1.

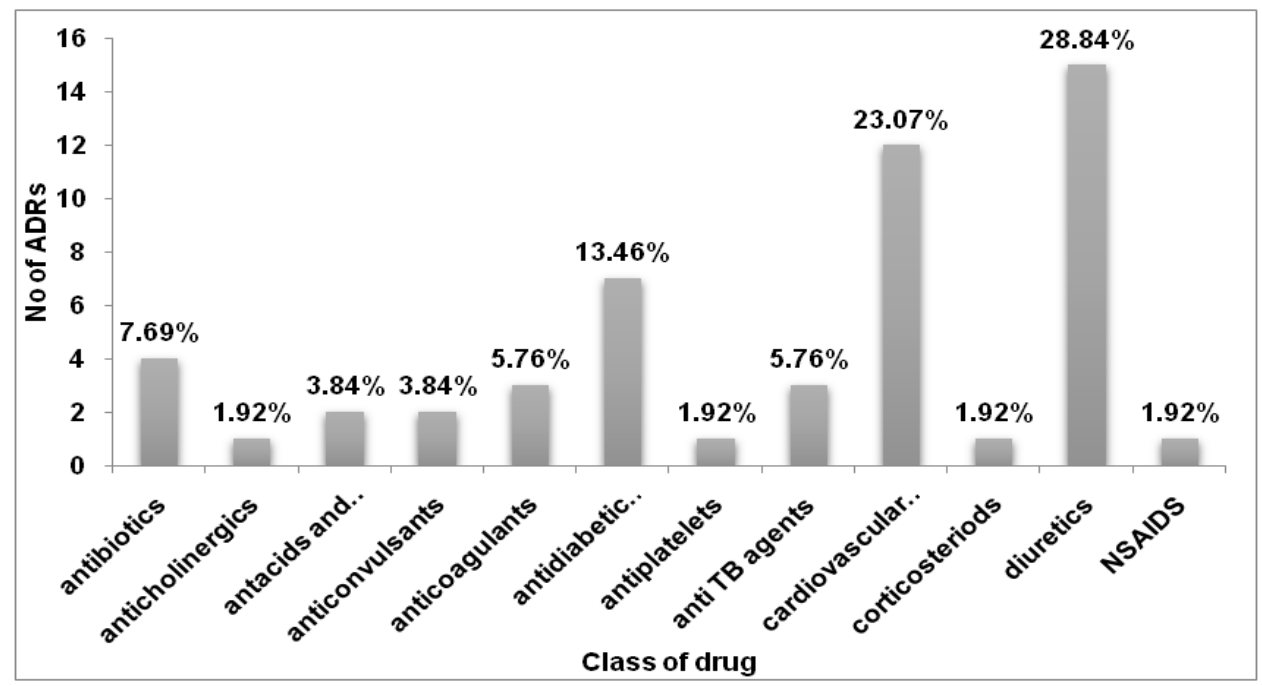

Fig. 3: Class wise distribution of ADRs

Out of 58 medication errors, 37(63.79\%) were identified in males and 21 $(36.20 \%)$ were identified in females. The most vulnerable age group that is prone to ME were 41-50 y age group of patients. A total of 190 cases were categorised according to types of ME. The commonly seen MEs are prescribing error, dispensing error, wrong drug error, wrong dose error, omission error, wrong time error, and compliance error.

\section{Pharmacist intervention}

By performing the intervention, the pharmacist evaluation shows that out of 190 cases, 104 (54.73\%) are solved, $52(27.36 \%)$ are partially solved and $34(17.89 \%)$ cases are not solved. The outcome of pharmacist evaluation was shown in table 6 .

\section{Categorisation of medication error}

Table 5: Categorisation of medication error

\begin{tabular}{|c|c|c|c|c|c|c|c|c|c|c|c|}
\hline Type of error & $\mathbf{A}$ & $\mathbf{B}$ & $\mathbf{C}$ & D & $\mathbf{E}$ & $\mathbf{F}$ & G & $\mathbf{H}$ & I & Total & Percent \\
\hline Prescribing Error & 0 & 2 & 8 & 0 & 0 & 3 & 0 & 0 & 0 & 13 & 22.413 \\
\hline Dispensing Error & 0 & 0 & 2 & 0 & 0 & 0 & 0 & 0 & 0 & 2 & 3.448 \\
\hline Wrong Drug Error & 0 & 0 & 6 & 0 & 0 & 0 & 0 & 0 & 0 & 6 & 10.344 \\
\hline Wrong Route Error & 0 & 0 & 0 & 0 & 0 & 0 & 0 & 0 & 0 & 0 & 0 \\
\hline Wrong Dosage Form Error & 0 & 0 & 0 & 0 & 0 & 0 & 0 & 0 & 0 & 0 & 0 \\
\hline Wrong Dose Error & 0 & 5 & 7 & 0 & 0 & 0 & 0 & 0 & 0 & 12 & 20.689 \\
\hline Omission Error & 0 & 5 & 13 & 0 & 0 & 0 & 0 & 0 & 0 & 18 & 31.034 \\
\hline Wrong Drug preparation Error & 0 & 0 & 0 & 0 & 0 & 0 & 0 & 0 & 0 & 0 & 0 \\
\hline Wrong Drug Administration Technique Error & 0 & 0 & 0 & 0 & 0 & 0 & 0 & 0 & 0 & 0 & 0 \\
\hline Wrong Time Error & 0 & 1 & 0 & 0 & 0 & 0 & 0 & 0 & 0 & 1 & 1.724 \\
\hline Wrong Patients & 0 & 0 & 0 & 0 & 0 & 0 & 0 & 0 & 0 & 0 & 0 \\
\hline Compliance Error & 0 & 2 & 4 & 0 & 0 & 0 & 0 & 0 & 0 & 6 & 10.344 \\
\hline Total & 0 & 15 & 40 & 0 & 0 & 3 & 0 & 0 & 0 & 58 & 100 \\
\hline
\end{tabular}

Table 6: Pharmacist intervention

\begin{tabular}{lll}
\hline Pharmacist evaluation & Frequency & Percentage \\
\hline Solved & 104 & 54.73 \\
Partially solved & 52 & 27.36 \\
Not solved & 34 & 17.89 \\
TOTAL & 190 & 100 \\
\hline
\end{tabular}

\section{DISCUSSION}

Polypharmacy was the frequent condition in Indian scenario and mainly depends upon the type of the diseases, comorbid conditions, hereditary, economic status and malnutrition.

Many medications have potential interactions with other drug or substance when prescribed together, according to the WHO definitions, the average number of drugs per person was within the range of 1.4-2.4. The present study found that an average number of drugs prescribed for patients was 9.82, showed the presence of polypharmacy.

Total of 1028 drug interactions was seen in 190 patients. The present study showed a prevalence of drug interactions were more in males 482(67.13) when compared to females 236(32.86).

Some other studies found a similar association which supports to 
our findings which were conducted by John et al. and mistry et al. [5].

In the present study maximum numbers of drug interactions were seen in 51-70 y age group. This result was in accordance with results of the study by the John et al. [5].

In this study, more number of drug interactions were observed in cardiology department 538 (52.33\%) in the previous study by g. Murtaza et al. showed the incidence of drug interactions were high in cardiology department[6]. In contrast to the study by John et al. showed less number of drug interactions in cardiology department [5].

The present study showed prescriptions which contain more than 6 drugs were predominately high $174(91.57 \%)$ which was similar to the study conducted by Ashok et al. [7].

The study conducted by kumara Swamy Rc et al. showed the average number of drugs per individual patient was 5.95 the present study showed that the average number of drugs per individual patient was 9.82[8]. Thus the increasing number of drugs increases the risk of occurrence of drug-drug interactions, ADRs exponentially (Gunasekaran et al.) [9].

Most of the drug interactions in our study were of major drug interactions 488 (47.47\%) while the other study performed by John et al. showed most of the drug interactions were moderate [5].

The present studies showed that high numbers of drug interactions were seen due to cardiovascular drugs $13(13.13 \%)$. the study which supports our findings was a study conducted by G. Murtaza et al. [6].

Most of the interactions in the present study were pharmacodynamics interactions $409(56.96 \%)$ were followed by pharmacokinetic interactions $309(43.04 \%)$ which was supported by the study performed by Kapadia et al. [10].

A total of 190 patients have included in the study out of them 43 patients developed 52 ADRs, out of which 36(69.23\%) ADRs were identified in male and $16(30.76 \%)$ ADRs were identified in females. In the previous study by M Sureswara reddy et al., showed the high percentage of ADRs in male [11]. A study by Desai et al., revealed a high percentage of ADRs in male [12]. Another study conducted by Jayanthi et al., showed male predominance [13].

The present study showed that 61-70 y age group patients were more prone to ADRs 15(28.845). these results are in accordance with the results of the study by Jayanthi et al.,which revealed a higher incidence of ADRs in patients age greater than 60 [13].

In the present study severity assessment is done by modified hart wig's scale showed $38(73.07 \%)$ moderate ADRs, $8(15.38 \%)$ severe ADRs and 6(11.53\%) mild ADRs were seen. A previous study by Madhavan trivedi et al., showed a high number of 34 moderate ADRs [14]. In contrast to the present study by Jayanthi et al. showed a high number of mild ADRs [13].

In this study, Causality assessment was done by using both Naranjo's scale and WHO-UMC scale. According to Naranjo's scales out of 52 ADRs10 (19.23\%) were definite, 10(19.23\%) ADRs were probable and 32(61.53\%) ADRS were possible. A study by Muthiah Palaniappan et al. showed that the majority of ADRs were possible and remaining ADRs were possible [15].

According to WHO-UMC scale out of 52 ADRs 6(11.53\%) ADRs were certain, $6(11.53 \%)$ ADRs were possible and 40(76.925) ADRs were probable. A study by Jayanthi et al. showed the most of the ADRs were probable [13].

In the present study, the highest number of ADRs were due to diuretics $15(28.84 \%)$ followed by cardiovascular drugs $12(23.07 \%)$. In contrast to the present study by M Sureswara Reddy et al., showed the highest number of ADRs in cardiovascular drugs followed by diuretics [11].

One fatal ADR was observed during the study period. Symptomatic treatment was given for 22(42.30\%) ADRs, a drug withdrawn are alternative treatment was given for $21(42.30 \%)$ ADRs and no change in the treatment was made for $9(17.30 \%)$ ADRs in the study.
These results are in accordance with the interventions of the study by Sagar B Bhagat et al. [16].

In the present study more number of omission errors $18(31.03 \%)$ and most commonly seen the category of medication errors were type C followed by type B. the study performed by the Syed Umer Jan et al., showed the high percentage of omission errors which was similar to our study [17]. Another study conducted by Hou Ning et al., showed more number of type B errors followed by type $\mathrm{C}$ errors which were the contrast to our study [18].

\section{Limitations of the study}

The current study evaluated the occurrence of DIs, ADRs and medication error at a single hospital that may not occur in another hospital, so a pilot study needs to be conducted throughout the country. Since DIs and ADRs can differ between patients based on disease condition and genetic characteristics. There is a possibility that many ADRs could have been unrecognized or unreported in the study. This study has a limitation of being a short term study which yields 52 ADRs The complete causality assessment could not be done due to not practicing re-challenge (not possible or not performed) for most of the cases. We probably failed to detect some errors, particularly administration errors. More reliably by trained observers following nurses during routine patient care activities

\section{CONCLUSION}

Finally, the study concluded that through multiple medications are seen in prescriptions can be accepted as they are appropriate to the present clinical condition but monitoring was required for the occurrence of drug-related problems. Hence Clinical pharmacist plays a potential role in the health care system in assisting the physician i.e. modifying the number of drugs taken, number of doses taken, medication adherence, identification of drug interactions, preventing, monitoring and detection of ADRs and identifying the medication errors. This will improve the patient quality of life and decreasing the health care costs of patients. So the Clinical pharmacist intervention plays a major role in both humanistic and clinical outcomes along with economic outcomes.

\section{ACKNOWLEDGMENT}

We are immensely grateful to our college, principal, parents, friends, and department of pharmacy practice for their valuable cooperation throughout the study for its successful completion and also patients attending to Vishwa Bharathi multispeciality hospitals, Kurnool, Andhra Pradesh, India for giving us their informed consent to use their case details for our study.

\section{AUTHORS CONTRIBUTIONS}

Dr Siddarama: Helped in data interpretation and manuscript evaluation, J Bharath Naidu: Development of complete research plan, K P Joshisree: Preparation and Drafting manuscript, V Sahithi Lakshmi: Helped to edit the complete manuscript all tables and fig. preparation, All the authors equally contributed to drafting the paper. All authors have read and approved the final manuscript.

\section{CONFLICT OF INTERESTS}

All authors have none to declare

\section{REFERENCES}

1. Mahesh Kumar VP, Dhanpal CK. Prevalence of polypharmacy in geriatric patients in the rural teaching hospital. Am J Phytomed Clin Ther 2014;2:413-9.

2. Acurcio FA. Analysis of medical prescriptions dispensed at health centers in Belo Horizonte, Minas Gerais, and Brazil. Cad Saude Publica 2004;20:72-9.

3. Alomar MJ. Factors affecting the development of adverse drug reactions. Saudi Pharm J 2014;22:83-94.

4. Akazawa M, Imai H, Igarashi A, Tsutani K. Potentially inappropriate medication use in elderly Japanese patients. Am J Geriatr Pharmacother 2010;8:146-60.

5. John NN, Udupi RH, Binu KM. Incidence of polypharmacy induced drug interaction in a tertiary care hospital. Int J Pharm Sci Res 2012;3:2119-21. 
6. Ghulam Murtasa. Assessment of potential drug-drug interactions and its associated factors in the hospitalized cardiac patients. Saudi Pharma J 2015;24:220-5.

7. Kumara Swamy RC. Prevalence of polypharmacy and drug to drug interactions in tertiary care teaching hospitals. Int Res J Pharm 2014;5:778-82.

8. Thirumurugan Gunasekaran, Natsanet Dejene, Vanapalli V Satyaveni, Magharla Dasaratha Dhanaraju. An occurrence of drug-drug interactions in Adama Referral Hospital, Adama city, Ethiopia. J Drug Assessment 2015;4:19-23.

9. Jigar Kapadia, Dhaval Thakor, Chetna Desai, RK Dikshit. A study of potential drug-drug interactions in indoor patients of the medicine department at a tertiary care hospital. J Appl Pharm Sci 2013;3:89-96.

10. M Sureshwara Reddy. Assessment, monitoring and reporting of ADRs due to polypharmacy. J Med Sci Clin Res 2016;4:1492631.

11. Jayesh M Kathiria, Bhagya M Sattigeri, Parth M Desai, Sumit $P$ Patel. A study of ADRs in patients admitted of intensive care unit of teaching rural hospitals. Int J Pharm Pharm Sci 2012;5:160-3.
12. Jayanthi. An observational study to analysis the ADR among the elderly at a tertiary care hospital. Biomed Pharma J 2017;10:345-52.

13. Trivedi M, Acharya T, Kotadiya F, Vekariya R, Mehta D. Pharmacovigilance study of beta-adrenergic receptor antagonists in patients visiting the department of medicine of a tertiary care hospital, Surendranagar, Gujarat, India. Int J Basic Clin Pharmacol 2016;5:2332-5.

14. Palaniappan M, Selvarajan S, George M, Subramaniyan G, Dkhar AS, Pillai AA, et al. Pattren of drug reactions reported with cardiovascular drugs in a tertiary care teaching hospital. J Clin Diagnostic Res 2015;9:1-4

15. Bhagat BS, Joshi DA, Patil CK, Gambre SR, Patel BS. Adverse drug reactions of anti-hypertensive in medicine department of tertiary care hospital: a twelve months observation. $\mathrm{Br}$ J Pharma Res 2015;6:166-73.

16. Syed Umer Jan, Rahman Gul. An assessment of rational drug use and evaluation of medication error in public sector hospitals. Res Gate 2016;19:A275.

17. Hou Ning, Tang Hui. Analysis of medication errors in the provincial hospital. Biomed J Sci Tech Res 2017;4:962-6. 